Computing and Informatics, Vol. 39, 2020, 340 360 doi: 10.31577/cai_2020_1-2 340

\title{
AN IMPROVED PDR LOCALIZATION ALGORITHM BASED ON PARTICLE FILTER
}

\author{
Wei Wang*, Cunhua Wang, Zhaoba Wang, Xiaoqian ZhaO \\ School of Information Science and Engineering \\ North University of China, Taiyuan, 030051, China \\ e-mail: 41695559@qq.com
}

\begin{abstract}
Pedestrian Dead Reckoning (PDR) helps to realize step frequency detection, step estimation and direction estimation through data collected by inertial sensors such as accelerometer, gyroscope, magnetometer, etc. The initial positioning information is used to calculate the position of pedestrians at any time, which can be applied to indoor positioning technology researching. In order to improve the position accuracy of pedestrian track estimation, this paper improves the step frequency detection, step size estimation and direction detection in PDR, and proposes a particle swarm optimization particle filter (PSO-IPF) PDR location algorithm. Using the built-in accelerometer information of the smartphone to carry out the step frequency detection, the step frequency parameter construction model is introduced to carry out the step estimation, the direction estimation is performed by the Kalman filter fusion gyroscope and the magnetometer information, and the positioning data is merged by using the particle filter. The fitness function in the particle swarm optimization process is changed in the localization algorithm to improve particle diversity and position estimation. The experimental results show that the error rate of the improved step frequency detection method is reduced by about $2.1 \%$ compared with the traditional method. The angle accuracy of the direction estimation is about $4.12^{\circ}$ higher than the traditional method. The overall positioning accuracy is improved.
\end{abstract}

Keywords: Indoor positioning, PDR, particle filtering, particle swarm optimization, data fusion

Mathematics Subject Classification 2010: 68W40

* Corresponding author 


\section{INTRODUCTION}

In today's world, information technology is developing rapidly. With the in-depth development of the Internet, technologies such as the Internet and $5 \mathrm{G}$ are gradually maturing, and location-based services will play an increasingly important role. As an application based on location services, indoor positioning gradually penetrates into all aspects of social life. Currently the Global Positioning Systems (GPS) and the BeiDou Navigation Satellite System (BDS) are widely used in the United States. These systems have been able to provide users with higher-precision outdoor positioning, such as on-board map navigation, which makes people's needs for outdoor activities met. However, due to a small spatial pattern of the interior, the indoor positioning has stricter requirements on accuracy. As for indoors signals, outside signals are easily blocked, attenuated or reflected. Therefore, some problems exist in the outdoor positioning system, such as insufficient accuracy and signal instability in indoor positioning, resulting in relatively poor reliability, continuity and stability of indoor positioning. It is important to develop indoor positioning technology to provide high-precision, convenience and mature indoor location services [1.

Currently, as smartphones are widely embedded with magnetometers, accelerometers, gyroscopes and other sensors, it is possible for smartphone-based pedestrian dead-reckoning (PDR) to adapt smartphones. PDR technology is mainly divided into three parts: step frequency detection, step estimation and direction estimation [2]. Poulose et al. [3] proposed a step frequency detection by combining the acceleration information and the gyro information. Wang [4 proposed a dynamic constrained gait detection method, which removed the interference caused by jitter, through using dynamic constraint amplitude and accelerating peak time. In step estimation, it often combined people's walking frequency and experience value to estimate. Manos et al. [5] estimated the direction angle by establishing a model of gravity-like direction. Kang et al. [6] established indoor positioning for pedestrians by establishing an inseparable walking mode and real-time deep learning network module. Lu et al. [7] proposed a new regression model using accelerating data to perform stride frequency detection. At the same time, it combined with map information and barometer for spatial three-dimensional positioning. Hasan and Mishuk [8] used Kalman filtering to fuse acceleration, gyroscope and magnetometer data for indoor positioning. Since most of the current smartphones have built-in sensors such as accelerometers, gyroscopes and magnetometers, and the accuracy is also higher and higher, it is possible to use the smartphone for PDR indoor positioning.

In this paper, the accelerometer and gyroscope data are filtered and pre-processed by FIR low-pass filter. Firstly, in step frequency detection, setting the time threshold, the acceleration threshold and the change of the state value respectively. Secondly, in step estimation, the steps are counted, and the step frequency parameters are introduced to estimate the step size to improve the accuracy of the estimation. Then the magnetometer and gyroscope data are fused by Kalman filter to estimate the direction. Finally, the particle filter is improved by changing the 
fitness function in the particle swarm optimization algorithm to improve particle diversity and the accuracy of indoor positioning.

The rest of the paper is organized as follows. Section 2 brings forward a few related works. Section 3 focuses on improved PDR algorithm, introduces the algorithm for step frequency detection, step size estimation and direction angle prediction as well as a new particle swarm optimization particle filter algorithm. Section 4 mainly verifies the advantages of the proposed algorithm in indoor positioning, which can improve the positioning accuracy, and Section 5 concludes the paper.

\section{PEDESTRIAN MOBILE SENSING TECHNOLOGY BASED ON INERTIAL SENSOR}

The most important technique for pedestrian motion perception in indoor positioning is track estimation. In indoor positioning, track estimation can be evolved into pedestrian trajectory estimation. Pedestrian dead reckoning mainly consists of three important parts: step detection, step estimation and direction estimation. The basis of the stride detection is that the pedestrian motion has periodic characteristics. The cycle of each step of the movement is from the beginning of a step to the end of a step. The output value of the acceleration sensor can visually see the waveform of the motion cycle. The fluctuation of the acceleration value is generally related to the height of the person and the individual, the exercise habits and road conditions. The step frequency of pedestrian movement can be obtained by analyzing the acceleration values.

The purpose of the stride detection is to detect whether a pedestrian has walked. When a person is walking with a smartphone, the horizontal acceleration and the vertical acceleration will exhibit periodic changes. Therefore, the pedestrian motion can be detected by periodically changing the acceleration in the walking motion. Figure 1 is an acceleration signal collected when the pedestrian is holding the smartphone while walking.

It can be seen from the figure that the acceleration in the $\mathrm{Z}$ direction is relatively obvious, the periodicity is better, the acceleration in the $\mathrm{X}$ and $\mathrm{Y}$ directions is weaker, and the change is not as obvious as the $\mathrm{Z}$ direction. Since the three-axis output component of the smartphone accelerometer is related to the attitude of the smartphone itself, in the actual situation, the hand-held smartphone has a certain randomness, and the result obtained directly by using the Z-axis output in some severe scenes will appear larger error. Therefore, in order to eliminate the acceleration signal fluctuation caused by the gesture of the smartphone itself, the three-axis output component of the acceleration information is modulo obtained to obtain the combined amount Zsyn.

$$
Z \operatorname{syn}=\sqrt{a_{x}^{2}+a_{y}^{2}+a_{z}^{2}}
$$

$a_{x}, a_{y}, a_{z}$ are the three-axis output components of the smartphone. Figure 2 is a comparison diagram of changes in acceleration information components and composite amounts while a person is walking with a mobile phone in his/her pocket. 


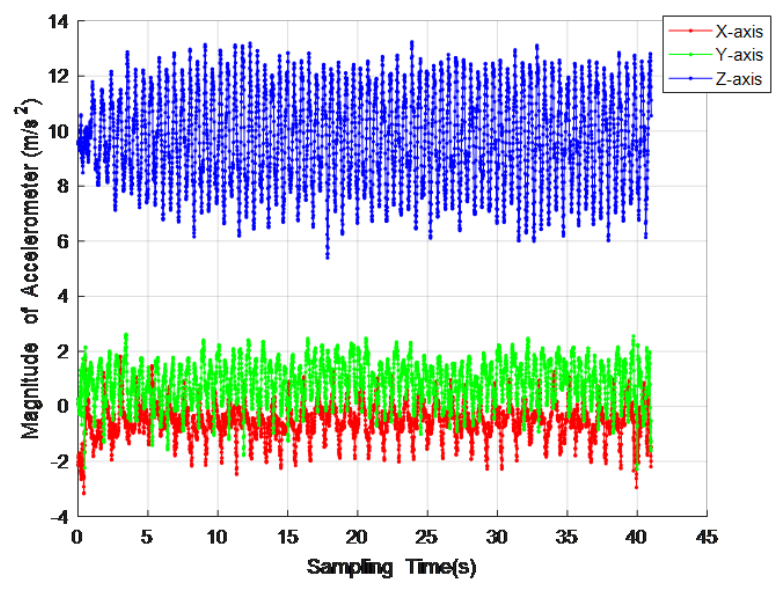

Figure 1. Change in acceleration value when walking

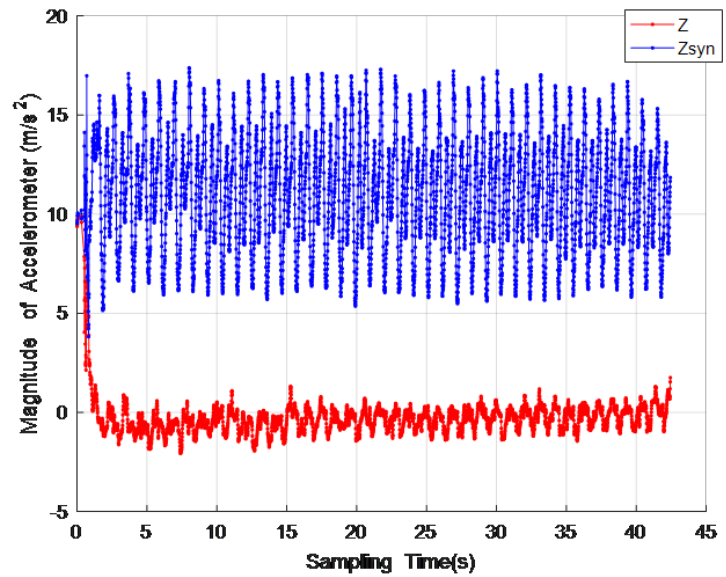

Figure 2. Comparison of dynamic Z component and composite quantity

It can be seen from Figure 2 that the Z-axis acceleration information fluctuation is not obvious when the smartphone is placed in the pocket. If the Z-axis acceleration information is also used to detect the pace, a large error will be caused, but the synthesis amount has a good periodicity. Therefore, the raw data of the acceleration information is used as the step to detect the original data. Moreover, the faster the walking speed from Figure 3, the larger the peak value and the valley value of the waveform. 


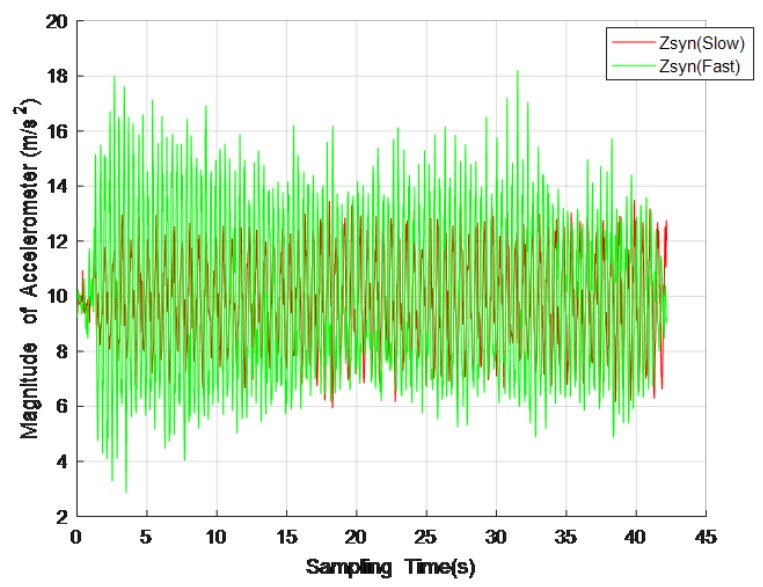

Figure 3. Acceleration waveform at different walking speeds

Considering that the human body is relatively fluctuating when walking, the collected acceleration information is mixed with random noise, so the acquired acceleration signal cannot be directly used, and it is necessary to perform related filtering processing to filter out large accidental noise and false peaks to obtain more obvious wave forms.

\section{PDR ALGORITHM}

\subsection{Step Frequency Detection}

The acceleration signal collected by the smartphone has high-frequency information interference, and it is particularly important to use the filter for filtering. Because the FIR filter has the advantages of good stability, high precision, small accumulation error, linear phase characteristics, etc., the acceleration signal is not easy to be distorted, so this paper selects the FIR filter to pre-filter the acceleration data.

The method of the digital filter design includes a window function method, a frequency sampling design method, and a multi-filter parallel processing method. In this paper, the window function method is selected to complete the filter design. The design idea is to choose an ideal frequency selection filter, which has an infinite impulse response and uses a suitable window function to cut off its impulse response to obtain a linear phase. The RF filter of the excited response is designed as follows:

$H_{d}\left(e^{j w}\right)$ represents an ideal low-pass filter and the ideal bandwidth filter is given by Formula (2):

$$
H_{d}\left(e^{j \omega}\right)= \begin{cases}e^{-j \omega}, & |\omega| \leq \omega_{c}, \\ 0, & \omega_{c}<|\omega| \leq \pi .\end{cases}
$$


In this formula, $\omega_{c}$ is the cutoff frequency, and the impulse response is:

$$
h_{d}(n)=\frac{1}{2 \pi} \int_{-\pi}^{\pi} H_{d}\left(e^{j w}\right) \mathrm{d} \omega=\frac{\sin \left[\omega_{c}(n-\alpha)\right]}{(n-\alpha) \pi} .
$$

In the formula: $\alpha=\frac{M-1}{2}$, in order to obtain an FIR filter from the ideal filter, it must be truncated by a windowing function to obtain a linear phase filter whose length is $M$ :

$$
h(n)= \begin{cases}h_{d}(n), & 0 \leq n \leq M-1, \\ 0, & \text { otherwise }\end{cases}
$$

The windowing function shows that $h(n)$ can be seen as the result of $h_{d}(n)$ multiplying by a window function:

$$
\begin{aligned}
& h(n)=h_{d}(n) \omega(n), \\
& \omega(n)= \begin{cases}0 \leq n \leq M-1, & \text { on the function of } \alpha \text {-symmetry } \\
0, & \text { otherwise }\end{cases}
\end{aligned}
$$

$h(n)$ is the filter required for the filter implementation. The filter order is $M$. At the same time, compared with the filters designed by different window functions, the Kaiser window not only has the lowest order, but also has a flat and minimum attenuation, so the Kaiser window is selected for design. After filtering and pre-processing the acceleration signal, the steps are counted by setting the time threshold, the acceleration threshold and the change of the state value. The specific algorithm steps are as follows:

- Set the status value to 0 .

- Read the acceleration data to determine whether the time threshold is met, and if so, continue the subsequent judgment, if not, continue to read the next acceleration data.

- Process the acceleration data to obtain a target value, determine whether the target value is greater than the acceleration threshold, and if greater than the threshold, set the state value to 1 , continue the third step of judgment, otherwise return to the first step.

- Determine whether the target value is greater than the current peak value. If it is greater, set the value to the new peak value, and then repeat the process until the maximum value is found, otherwise proceed to the next step.

- Determine whether the target value is less than zero. If it is less than 0 , set the status value to 2 , otherwise return to the first step.

- Determine whether the target value is less than the current minimum value, if it is less, set the value to the new minimum value, and then repeat the process until the minimum value is found, otherwise proceed to the next determination. 
- Determine whether the current target value is greater than 0 . If it is greater than 0 , it means that the step counting process is completed, otherwise return to the first step.

\subsection{Step Estimate}

Since different people's step sizes are different, individual differences can be reflected in the walking frequency of pedestrians. The model calculation formula is as follows:

$$
\text { stepLen }_{k}=(1-\eta)\left(0.56 \sqrt[2]{A C C_{p v}}+0.1 \mathrm{l} b\left(A C C_{p v}\right)\right)+\eta\left(a f^{2}+b f+c\right) .
$$

In Formula (6), $A C C_{p v}$ is the difference between the maximum and minimum values of acceleration in each step cycle; $a, b$ and $c$ are the step control parameters of the acceleration model, respectively, taken as $0.3,0.2$ and $0.1, \eta$ is step frequency weighting factors, taking $0.4, f$ is steps frequency. The selection of the experimental data is based on the step size of a large number of different people for statistical analysis, and then calculate the average.

\subsection{Direction Angle Estimation}

Since the carrier coordinate system of the mobile phone is different from the navigation coordinate system used in the actual positioning process, the sensor data is transformed between the two coordinate systems. The acceleration of the mobile phone and the sensor data acquired by the gyroscope are all for the carrier coordinate system. During the movement, the position change of the user is for the navigation coordinate system. The navigation coordinate system used in this paper takes the carrier centroid as the origin and the $\mathrm{X}$ axis along the West to the East, the $\mathrm{Y}$ axis is northward along the meridian, and they are all in the local horizontal plane. The $\mathrm{Z}$ coordinate axis is vertically upward along the local geographic vertical line, that is, the east-north-up geographic coordinate system. The coordinate system transformation can be realized by rotating the matrix. In this paper, the quaternion method is used for conversion. The gyro-based incremental rotation matrix based on the quaternion is shown in Formula (7):

$$
\Delta R=\left[\begin{array}{lll}
q_{0}^{2}+q_{1}^{2}-q_{2}^{2}-q_{3}^{2} & 2\left(q_{1} q_{2}-q_{0} q_{3}\right) & 2\left(q_{1} q_{3}+q_{0} q_{2}\right) \\
2\left(q_{1} q_{2}+q_{0} q_{3}\right) & 1-2\left(q_{1}^{2}+q_{3}^{2}\right) & 2\left(q_{2} q_{3}-q_{0} q_{1}\right) \\
2\left(q_{1} q_{3}-q_{0} q_{2}\right) & 2\left(q_{2} q_{3}+q_{0} q_{1}\right) & q_{0}^{2}-q_{1}^{2}-q_{2}^{2}+q_{3}^{2}
\end{array}\right]
$$


$q_{i}(i=1,2,3,4)$ corresponds to the four sub-elements in the quaternion and can be solved by Formula (8):

$$
\begin{aligned}
Q & =\left[q_{0}, q_{1}, q_{2}, q_{3}\right] \\
& =\left[\cos \frac{\theta}{2}, \sin \frac{\theta}{2}\left(\frac{\omega_{x}}{\sqrt{\omega_{x}^{2}+\omega_{y}^{2}+\omega_{z}^{2}}}, \frac{\omega_{y}}{\sqrt{\omega_{x}^{2}+\omega_{y}^{2}+\omega_{z}^{2}}}, \frac{\omega_{z}}{\sqrt{\omega_{x}^{2}+\omega_{y}^{2}+\omega_{z}^{2}}}\right)\right] .
\end{aligned}
$$

In Formula (8), $\omega_{x}, \omega_{y}, \omega_{z}$ are the output components of the smartphone in the carrier coordinates, $\theta$ is the angular velocity mode, $|\omega|$ is the angular velocity increment obtained by integrating the time. Since the gyroscope cannot obtain the initial direction, it is possible to obtain an initial rotation matrix Rc by the smartphone at rest and then multiply the incremental rotation matrix of the gyroscope with the previous matrix to obtain the rotation matrix of the gyroscope. The initial rotation matrix Rc can be obtained by the roll angle $\varphi$, pitch angle $\phi$ and heading angle $\theta$ at res.

Assume that the accelerometer measures the three-axis component of the acceleration: $a^{b}=\left[a_{x}, a_{y}, a_{z}\right]$ in the carrier coordinates. Under the navigation coordinate system, it is: $a^{n}=\left[a_{E}, a_{N}, a_{U}\right]$, we can get:

$$
a^{b}=R_{c} a^{n}
$$

In the static state, $a^{n}=[0,0, g]^{T}$, in which the local gravity acceleration is taken as 9.8. According to the attitude angle conversion formula, the formula can be obtained:

$$
\begin{aligned}
\theta & =\arctan \left(\frac{M_{x} \cos \phi+M_{z} \sin \phi}{\left(M_{x} \sin \phi+M_{z} \cos \phi\right) \sin \varphi+M_{y} \cos \varphi}\right), \\
{\left[\begin{array}{l}
a_{x} \\
a_{y} \\
a_{z}
\end{array}\right] } & =\left[\begin{array}{l}
-g \sin \varphi \cos \phi \\
g \sin \phi \\
g \cos \varphi \cos \phi
\end{array}\right] .
\end{aligned}
$$

According to Formula (10), the heading angle can be obtained, and the roll angle and the pitch angle can be obtained according to Formula (11). Then solve the four variables of the quaternion by the relationship between the quaternion and the attitude angle:

$$
\left[\begin{array}{l}
q_{0} \\
q_{1} \\
q_{2} \\
q_{3}
\end{array}\right]=\left[\begin{array}{c}
\cos \frac{\gamma}{2} \cos \frac{\theta}{2} \cos \frac{\varphi}{2}+\sin \frac{\gamma}{2} \sin \frac{\theta}{2} \sin \frac{\varphi}{2} \\
\sin \frac{\gamma}{2} \cos \frac{\theta}{2} \cos \frac{\varphi}{2}-\cos \frac{\gamma}{2} \sin \frac{\theta}{2} \sin \frac{\varphi}{2} \\
\cos \frac{\gamma}{2} \sin \frac{\theta}{2} \cos \frac{\varphi}{2}+\sin \frac{\gamma}{2} \cos \frac{\theta}{2} \sin \frac{\varphi}{2} \\
\cos \frac{\gamma}{2} \cos \frac{\theta}{2} \sin \frac{\varphi}{2}-\sin \frac{\gamma}{2} \sin \frac{\theta}{2} \sin \frac{\varphi}{2}
\end{array}\right] .
$$

After the initial rotation matrix is calculated, the incremental rotation matrix 
updated by the gyroscope is continuously multiplied by the previous rotation matrix to obtain the updated gyroscope rotation matrix.

$$
\begin{gathered}
R_{g}^{k}=R_{c} \prod_{i=0}^{k} \Delta R_{g}^{k}, \\
\left\{\begin{array}{c}
\theta=\arctan \left(\frac{T(2,2)}{T(1,2)}\right), \\
\varphi=\arcsin \left(-\frac{T(3,1)}{1}\right), \\
\phi=\arctan \left(-\frac{T(3,3)}{T(3,1)}\right) .
\end{array}\right.
\end{gathered}
$$

$\Delta R_{g}^{k}$ is the gyroscope incremental rotation matrix from the $k-1$ to the $k$ sampling; $R_{g}^{k}$ is the gyroscope rotation matrix incremented for the $k$ sampling. After obtaining the rotation matrix of the gyroscope $R_{g}^{k}$, the posture information in the navigation coordinate system can be solved. $T(i, j)$ represents the $i$ row and $j$ column elements in the rotation matrix.

Considering the complementary advantages of gyroscope and magnetometer information, Kalman filter can be used to fuse the two kinds of information. The specific fusion process is: assuming that $\theta_{k}$ is the estimated direction of the pedestrian at time $k ; \Delta \theta_{k}$ is the amount of change of the gyroscope at the time $k-1$ to $k$, then the system state equation is:

$$
S_{k}=A S_{k-1}+B \Delta S_{k}+C_{k}
$$

In the formula, $S_{k}=\left[\theta_{k}\right]$ is the system state, $A=B=[1]$ is the system parameter; $C_{k}$ is the system process noise, and the system observation variable $D_{k}$ is the magnetometer output, so the observation equation is:

$$
D_{k}=H S_{k}+\xi_{k}
$$

$H=[1]$ is the system parameter and $\xi_{k}$ is the noise the system observes, so the Kalman filter prediction and update process is as follows:

\section{Observation process:}

$$
\left\{\begin{array}{l}
S_{k \mid k-1}=A S_{k-1 \mid k-1}+B \Delta S_{k}, \\
P_{k \mid k-1}=A P_{k-1 \mid k-1} A^{T}+B \Delta \theta_{k}+C^{k} .
\end{array}\right.
$$

\section{Update process:}

$$
\left\{\begin{array}{l}
K_{k}=P_{k \mid k-1} H^{T}\left(H P_{k \mid k-1} H^{T}+\xi^{k}\right)^{-1} \\
S_{k \mid k}=S_{k \mid k-1}+K_{k} D_{k}-K_{k} H S_{k \mid k-1} \\
P_{k \mid k}=P_{k \mid k-1}-K_{k} H P_{k \mid k-1} .
\end{array}\right.
$$


$P_{k}$ is the system co-variance matrix; $K_{k}$ is the Kalman filter gain.

\section{4 (PSO-PF) Particle Swarm Optimization Particle Filter}

Traditional particle swarm optimization particle filter algorithm (PSO-PF) in the search field to fitness function as evaluation standard updates the size of the particle state change quantity, and then adjusts the spatial distribution of particles, the particle state concentrated in the fitness function extremum near. Filtering algorithm introduces the latest measurement values to the sampling distribution, makes particles move backward probabilistic higher area, and in the process of optimization iterations, because each particle state change amount of random variation, it ensures the diversity of particles, thus improves the particle degradation problems of particle filter. However, the fitness function used by this algorithm which selects the optimal particle is based on the difference between the current measured value and the predicted value of measured value as the evaluation standard. When the noise variance becomes high, it directly affects the selection of the optimal particle, resulting in a significant decrease in the filtering performance with the increase of noise variance.

The PSO-IPF algorithm proposes a new fitness function, which takes the difference between each particle state and state estimation as the evaluation standard, weakens the influence of random measurement noise and improves the filtering accuracy of the algorithm.

General dynamic time-varying systems can be described as 19$)$ and 20 :

$$
\begin{aligned}
& x_{k}=f\left(x_{k-1}\right)+v_{k} \sim p\left(x_{k} \mid x_{k-1}\right), \\
& z_{k}=h\left(x_{k}\right)+w_{k} \sim p\left(z_{k} \mid x_{k}\right) .
\end{aligned}
$$

$\mathrm{X}_{\mathrm{k}} \in \mathrm{R}^{\mathrm{n}}$ is the $n$-dimensional state measurement vector of the system at the time of $k ; \mathrm{Z}_{\mathrm{k}} \in \mathrm{R}^{1}$ is the $l$-dimensional measurement of the system at the time of $k, \mathrm{~V}_{\mathrm{k}} \in \mathrm{R}^{\mathrm{a}}, \quad \mathrm{W}_{\mathrm{k}} \in \mathrm{R}^{1}$ are process noise and measurement noise, respectively. The algorithm takes the particle prior probability as the importance function, calculates the corresponding particle weight by extracting the particle sample, and then obtains the weight to normalize, and finally obtains the weighted particle set. After resampling, the particle set is $\left\{x_{k}^{i}, 1 / N\right\}_{i=1}^{N}$.

In the optimization process, the particle state set is taken as the initial state of the particle swarm optimization, $x_{p}^{i}(m)$ and $x_{g}(m)$ are the individual optimal value and the local optimal value of the $m^{\text {th }}$ iterative particle state, respectively, and finally according to the particle fitness function (21) to choose. In the formula, $\sigma^{2}$ is the measuring noise variance, and $z_{\text {pre }}=h\left(x_{k}^{i}(m-1)\right)$ is the predicted value.

$$
s_{k}^{i}(m-1)=\exp \left[-\frac{1}{2 \sigma^{2}}\left(z_{k}-z_{\text {pre }}\right)^{2}\right] .
$$

In the $m$ times iteration, if the value of the $n^{\text {th }}$ particle fitness function is larger, 
then the current state of the particle is updated to its individual optimal value, otherwise the result of the last iteration is retained. Then, the model is updated according to the state variable, and the particle state change is calculated as follows:

$$
\begin{aligned}
v_{x}^{i}(m) & =\omega v_{x}^{i}(m-1)+\varphi_{1}\left(x_{p}^{i}(m-1)-x_{k}^{i}(m-1)\right)+\varphi_{2}\left(x_{g}(m-1)-x_{k}^{i}(m-1)\right), \\
m & =1,2, \ldots, D .
\end{aligned}
$$

In Formula (22), $\omega$ is the inertia weight factor, $V_{x}^{i}(m)$ is the uniform distribution of $[0,1], \varphi_{1}: \varphi_{2}$ is the random number of $[0,1]$, and $\mathrm{D}$ is the total number of iterations. The updated particle state value is:

$$
x_{k}^{i}(m)=x_{k}^{i}(m-1)+v_{x}^{i}(m) .
$$

Although this algorithm improves the particle degradation problem and maintains the particle diversity and improves the filtering accuracy, the fitness function is based on the difference between the current measured value and the measured predicted value as the evaluation standard, which is largely affected by the measurement noise, causing lower filtering accuracy. Therefore, the filter value (state estimation value) which is less affected by the noise variance is selected as the reference value of the selected particle, and the improved fitness function expression is in Formula 24.

$$
s_{k}^{i}(m)=\exp \left(-\frac{1}{C}\left(x_{k}^{i}(m)-\underset{k}{\stackrel{\wedge}{x}}\right)^{2}\right) .
$$

In Formula (24), $x_{k}^{i}(m)$ is the state value of the $m^{\text {th }}$ iteration of the $i^{\text {th }}$ particle; $x_{k}^{\wedge}$ is the state estimation value of the pre-optimization particle filter, and $C$ is the constant selected according to the convergence of the iteration.

The algorithm steps are as follows:

Step 1: Importance sampling process. According to the system model, obtain particles $\left\{x_{k}^{i} \mid x_{k}^{i} \sim p\left(x_{k}^{i} \mid x_{k-1}^{i}\right)\right\}$, and then obtain the normalized weight of the particle filter part of the particle, and finally get the weighted particle set $\left\{x_{k}^{i}, w_{k}^{i}\right\}_{i=1}^{N}$.

Step 2: The initial state estimation process. The state estimation of the particle filtering part is performed according to Formula (25), the initial filtering result $x_{k}^{\wedge}$ is obtained, and the estimation result is taken as an important parameter of the fitness function in the optimization step.

$$
\underset{k}{\stackrel{x}{x}}=\sum_{i=1}^{N} w_{k}^{i} x_{k}^{i} .
$$

Step 3: PSO processing is divided into three steps:

1. Initialize the population, and use the particles obtained by the current particle filtering as the initial population $\left\{x_{k}^{i}(0), w_{k}^{i}\right\}_{i=1}^{N}=\left\{x_{k}^{i}, w_{k}^{i}\right\}_{i=1}^{N}$. 
2. Calculate the fitness function and update the individual optimal value and the global optimal value by calculating the fitness function of each particle.

- If $s_{k}^{i}(m) \geq s_{k}^{i}(m-1)$, then $x_{p}^{i}(m)=x_{k}^{i}(m)$.

- If $s_{k}^{i}<s_{k}^{i}(m-1)$, then $x_{p}^{i}(m)=x_{p}^{i}(m-1)$. Find the maximum particle number $I(m)$ in the $s_{k}^{i}(m)$.

- If $s_{k}^{I(m)}(m) \geq s_{k}^{I(m-1)}(m-1)$, then $x_{g}(m)=x_{k}^{I(m)}(m)$. If $s_{k}^{I(m)}(m)<$ $s_{k}^{I(m-1)}(m-1)$, then $x_{g}(m)=x_{g}(m-1)$.

3. Adjust the state of the particles, and update the position and velocity of the particles to obtain the state change $v_{x}^{i}(m+1)$ of the $(m+1)^{\text {th }}$ iteration of each particle and the state $x_{k}^{i}(m+1), i=1,2, \ldots, N$.

4. Return to step 2 until the optimization is complete.

Step 4: Output state estimation: Select the final global optimal value of the particle $\widehat{x}_{k}=x_{g}(D)$ as the exact filtered output.

In order to verify the filtering effect of the PSO-IPF algorithm proposed in this paper, the nonlinear non-Gaussian system model is filtered by the algorithm, standard PF algorithm and PSO-PF algorithm, respectively.

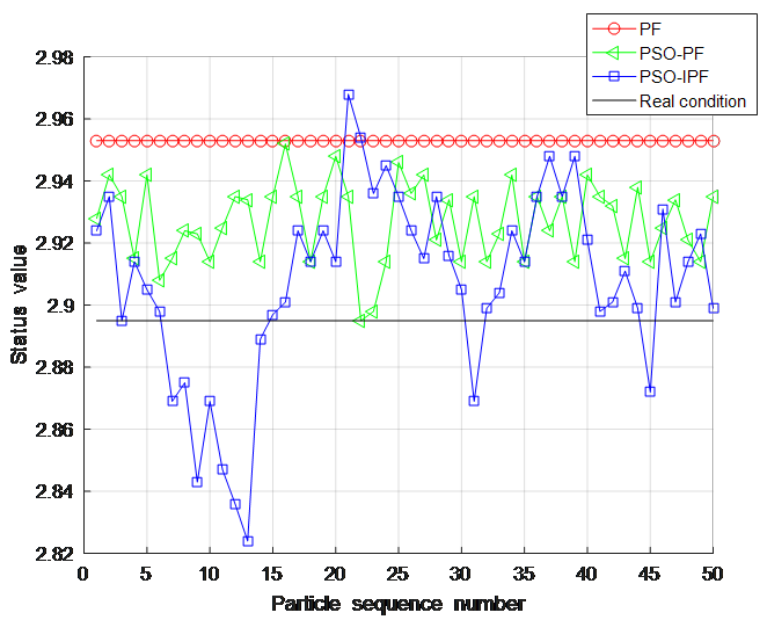

Figure 4. The particle distribution of the three algorithms $(k=20)$

It can be seen from Figure 4 that at the time of $k=20$, the particle state of $\mathrm{PF}$ is unique, which is due to the serious loss of particle diversity caused by re-sampling, while PSO-PF and PSO-IPF can ensure the diversity of particles. In contrast, PSO-IPF algorithm has the best results. 


\section{EXPERIMENTAL VERIFICATION}

The experimental site was held on the third floor corridor of the Science Building of North University of China. The total length of the route in the corridor is $40 \mathrm{~m}$, and the width of the corridor is $1.6 \mathrm{~m}$. The plan view is shown in Figure 5 The size of the floor tile $(0.8 \mathrm{~m} \times 0.8 \mathrm{~m})$ divides the experimental area into 100 grid cells of the same size, and the vertices of each grid cell serve as sampling reference points for a total of 303 reference points.

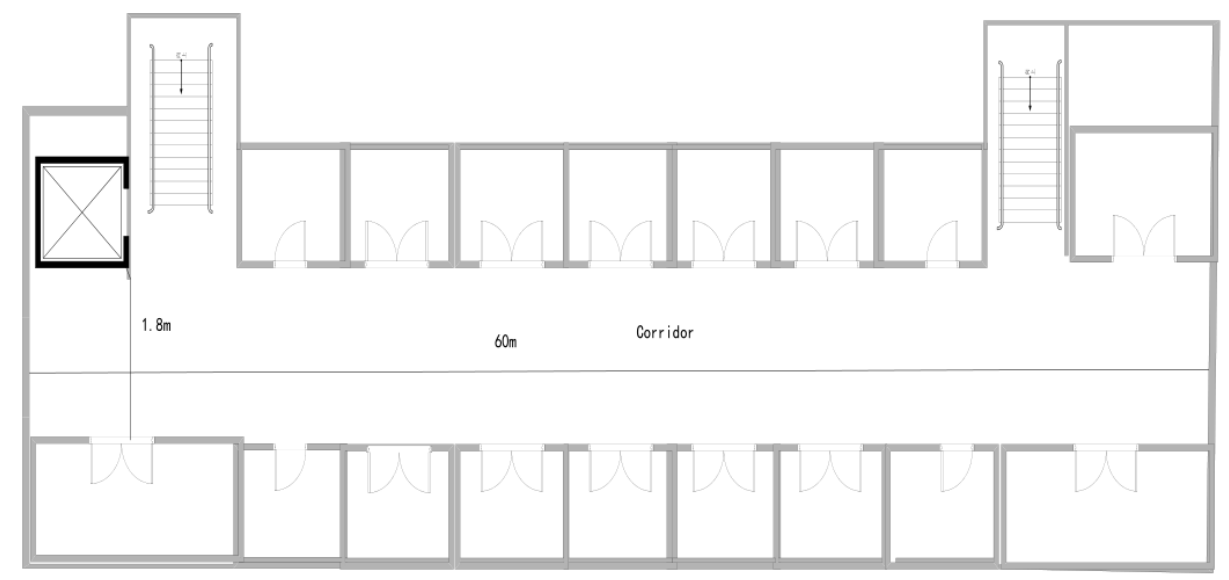

Figure 5. A plan view

The mobile phone used in this article is equipped with a three-axis magnetometer model AK09915 manufactured by AKM. The magnetometer has a measurement accuracy of 0.12856238 and is equipped with sensors such as the LSM6DS3 accelerometer and the LSM6DS3 gyroscope. Set the mark on the ground to record the pedestrian walking process, and use the smartphone to collect pedestrian walking data, collect acceleration, gyroscope and magnetometer data through the mobile phone, and set the sampling frequency to $50 \mathrm{~Hz}$; the experimental scene and mobile phone collection information are shown in Figures 6 and 7.

\subsection{Step Frequency Test}

In order to verify the accuracy of the step detection, the experiment chose to allow three experimenters with different heights and weights to carry the same smartphone to walk in the same experimental environment. Each person made three experiments to record the actual number of steps and walking of the experimenter. Acceleration sensor data in the process, for the reliability of the result, each person's route is different each time. Then after the collected original acceleration data is subjected to FIR low-pass filtering, the step frequency detection method and the traditional peak 


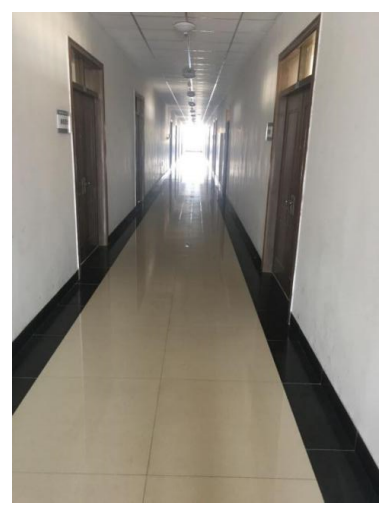

Figure 6. Experimental scene diagram

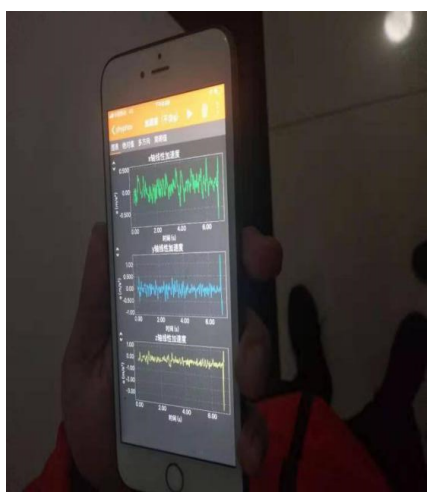

Figure 7. Smartphone data collection site map

detection method of this article are used. The zero detection method is compared, and the experimental results are shown in Table 1 .

It can be seen from Table 1 that the accuracy of our algorithm in 9 groups experiments is:

$$
1-\frac{0+2+2+1+3+3+1+2+3}{63+69+80+71+85+79+72+79+83} \times 100 \%=97.6 \% .
$$

The accuracy of the peak detection in the experiments is:

$$
1-\frac{4+4+3+4+3+10+4+7+10}{63+69+80+71+85+79+72+79+83} \times 100 \%=92.9 \% .
$$

The accuracy of the zero-crossing detection in the experiments is:

$$
1-\frac{3+2+3+3+1+5+3+4+6}{63+69+80+71+85+79+72+79+83} \times 100 \%=95.5 \% .
$$




\begin{tabular}{|c|c|c|c|c|c|c|c|c|}
\hline $\begin{array}{l}\text { Experi- } \\
\text { ment }\end{array}$ & Group & $\begin{array}{l}\text { Real } \\
\text { Step }\end{array}$ & $\begin{array}{r}\mathrm{Ca} \\
\text { Peak } \\
\text { Detection }\end{array}$ & $\begin{array}{l}\text { lculation St } \\
\text { Zero-Cross } \\
\text { Detection }\end{array}$ & $\begin{array}{l}\text { ep } \\
\text { Improved } \\
\text { Method }\end{array}$ & $\begin{array}{r}\text { Peak } \\
\text { Detection }\end{array}$ & $\begin{array}{r}\text { Error [Step] } \\
\text { Zero-Cross } \\
\text { Detection }\end{array}$ & $\begin{array}{r}\text { Improved } \\
\text { Method }\end{array}$ \\
\hline Tester 1 & 1 & 63 & 67 & 66 & 63 & 4 & 3 & 0 \\
\hline & 2 & 69 & 73 & 71 & 69 & 4 & 2 & 2 \\
\hline & 3 & 80 & 83 & 83 & 81 & 3 & 3 & 2 \\
\hline Tester 2 & $\overline{4}$ & 71 & 75 & 74 & 72 & 4 & 3 & 1 \\
\hline & 5 & 85 & 88 & 86 & 85 & 3 & 1 & 3 \\
\hline & 6 & 79 & 89 & 84 & 77 & 10 & 5 & 3 \\
\hline Tester 3 & 7 & 72 & 76 & 75 & 72 & 4 & 3 & 1 \\
\hline & 8 & 79 & 86 & 83 & 78 & 7 & 4 & 2 \\
\hline & 9 & 83 & 93 & 89 & 86 & 10 & 6 & 3 \\
\hline
\end{tabular}

Table 1. Analysis of the results of the step detection test

Figure 8 is an acceleration detection waveform diagram using the method herein.

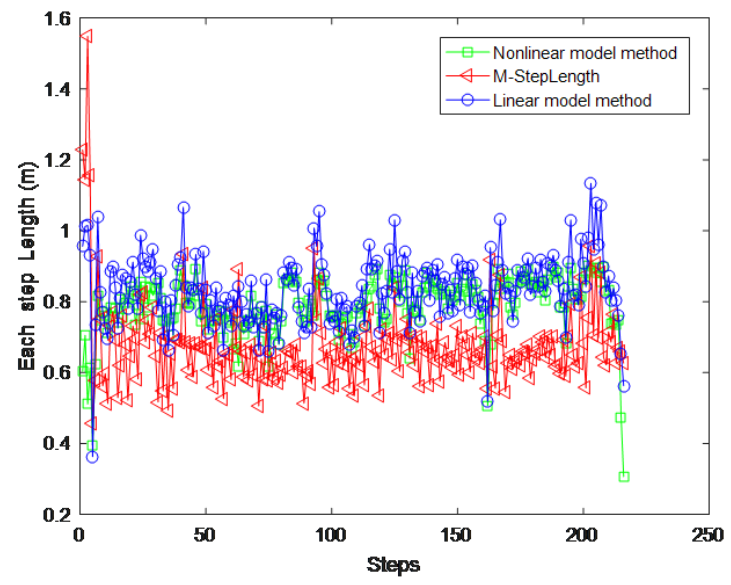

Figure 8. Step detection waveform and step count statistics

\subsection{Pedestrian Step Estimation Experiment}

In order to verify the accuracy of the step estimation method, experiments were carried out on the corridor, and the data collected by the experiment was compared with the conventional nonlinear model by using the step size and linear model calculated by the method. Figure 9 compares the three different methods of step size calculation, Figure 10 shows the average error of each step (the absolute value of the step difference between each step and the measured average). 


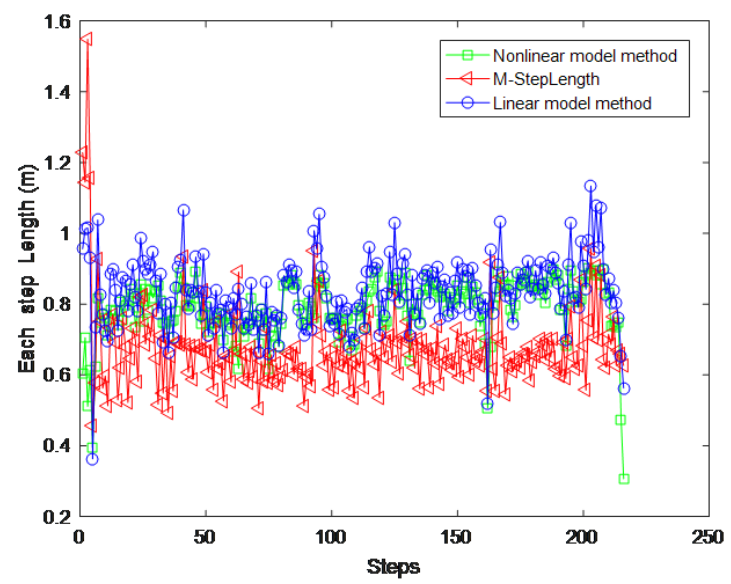

Figure 9. Comparison of three methods of step size calculation

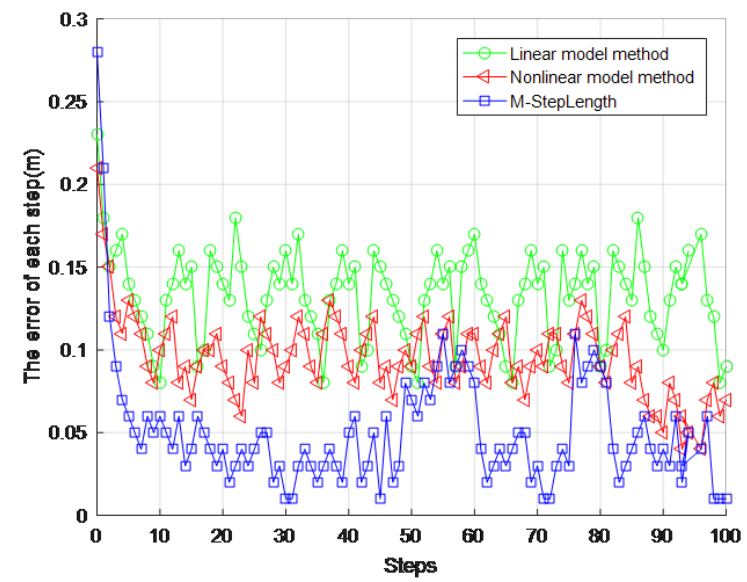

Figure 10. Average error per step

It can be seen from Figure 9 that the step sizes calculated by the method are distributed between $0.5 \mathrm{~m}$ and $0.8 \mathrm{~m}$, which is in accordance with the actual value of the walking step of the person. As can be seen from Figure 10, the error of each step of the method is relatively small, and is distributed between $0 \mathrm{~m}$ and $0.1 \mathrm{~m}$. Considering this, the method has certain advantages. 


\subsection{Direction Angle Estimation Experiment}

In order to verify the effectiveness of the direction estimation algorithm, a path that changes direction is selected in the laboratory environment to verify the validity of the method estimation. The gyro and magnetometer data collected by the smartphone are used for Kalman filter fusion and localization, and compared with the method of using the gyroscope and the magnetometer alone. Figure 11 presents a direction angle estimation value obtained by different methods, and Figure 12 shows a direction estimation cumulative error probability distribution curve.

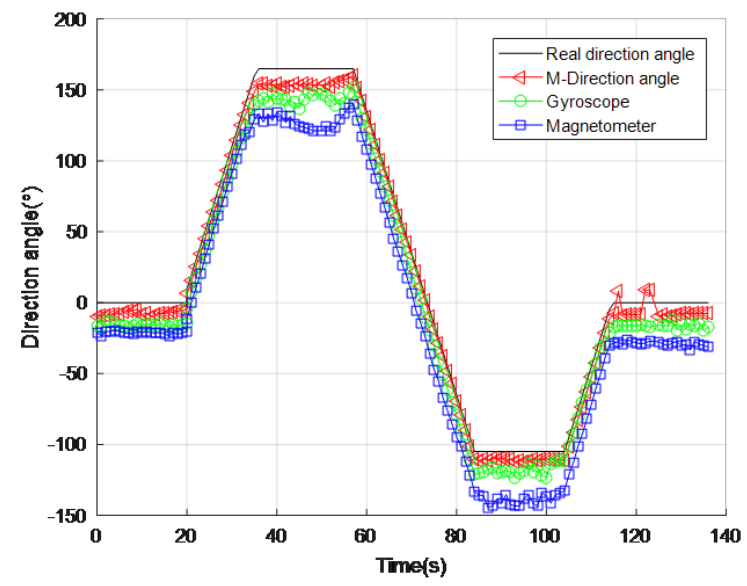

Figure 11. Comparison of different method direction angles

It can be seen from Figure 11 that the direction angle estimation method proposed in this paper is applicable to the process of changing direction. At the same time, it can be seen from Figure 12 that the cumulative probability distribution of the estimation method error within 5 degrees is 0.78 , which is significantly higher than the other two. 0.52 using of the gyroscope alone and 0.29 using the magnetometer alone, the cumulative probability distribution with an error of less than 10 degrees is 0.91 , which is significantly higher than 0.81 using the gyroscope alone and 0.65 using the magnetometer alone.

\subsection{PDR Indoor Positioning Experiment}

In order to verify the positioning of the improved PDR positioning algorithm in indoor positioning, two different experiments were performed in the laboratory environment. Figure 13 shows the positioning of the experimenter's rectangular path, and Figure 14 presents the positioning of the experimenter's straight path.

It can be seen from Figures 13 and 14 that whether the pedestrian walks a rectangular path or a straight path, the improved PDR localization algorithm is improved 


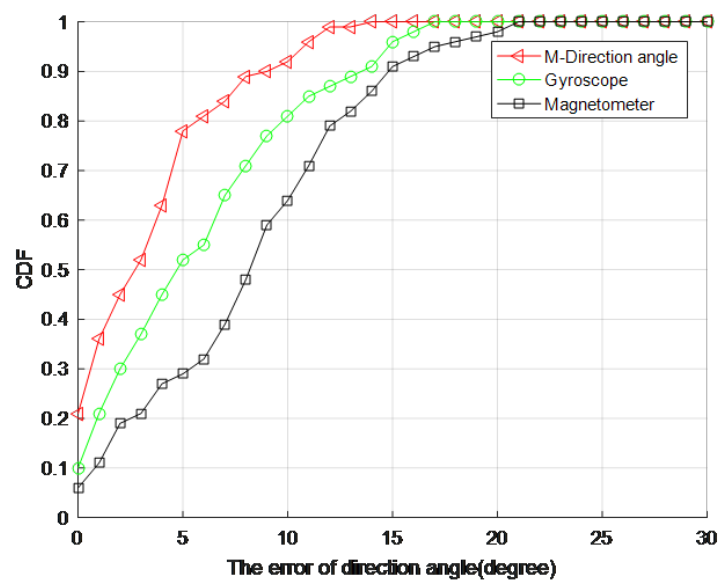

Figure 12. Directional estimation cumulative error probability distribution curve

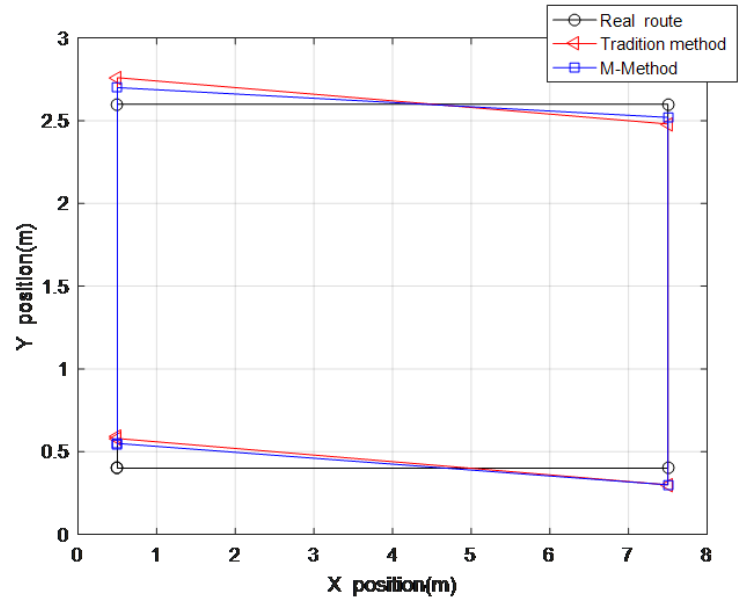

Figure 13. Position trajectory of rectangular path

compared with the traditional PDR localization algorithm, and the positioning accuracy is also improved.

\section{CONCLUSION}

In order to increase the indoor positioning accuracy of PDR, this paper firstly improves the step frequency detection, step size estimation and direction angle estimation in PDR algorithm, and then passes the problem of particle diversity weakening 


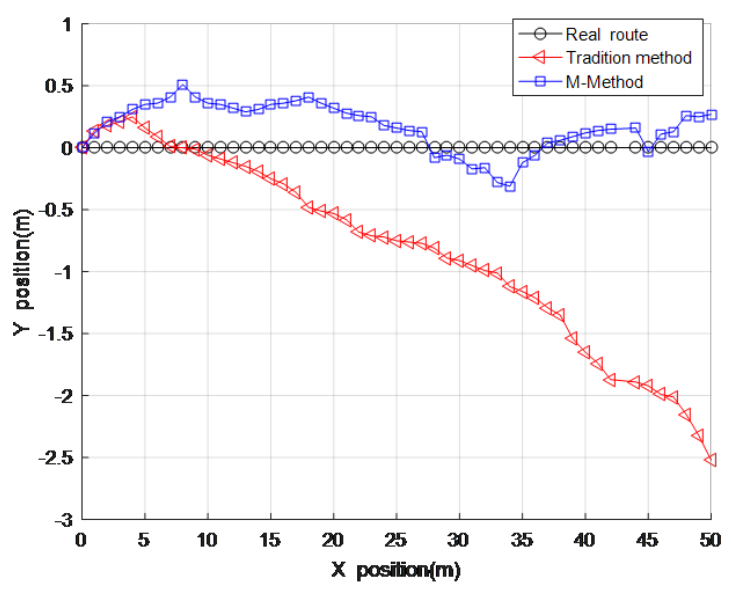

Figure 14. Position trajectory of straight path

over time in the particle filtering process. The fitness function in the particle swarm optimization algorithm is modified to optimize the particle filter to increase particle diversity and improve positioning accuracy.

\section{REFERENCES}

[1] Maghdid, H. S.-Lami, I. A.-Ghafoor, K. Z.-Lloret, J.: Seamless Outdoors-Indoors Localization Solutions on Smartphones: Implementation and Challenges. ACM Computing Surveys, Vol. 48, 2016, No. 4, Art. No. 53, 34 pp., doi: $10.1145 / 2871166$.

[2] Li, N.-Chen, J.-Yuan, Y.-Song, C.: A Fast Indoor Tracking Algorithm Based on Particle Filter and Improved Fingerprinting. $201635^{\text {th }}$ Chinese Control Conference (CCC), Chengdu, China, IEEE, 2016, pp. 5468-5472, doi: 10.1109/ChiCC.2016.7554206.

[3] Poulose, A.-Eyobu, O.S.-Han, D.S.: An Indoor Position-Estimation Algorithm Using Smartphone IMU Sensor Data. IEEE Access, Vol. 7, 2019, pp. 11165-11175, doi: 10.1109/ACCESS.2019.2891942.

[4] Wang, Z.: Research on Indoor Location Algorithm Based on Inertial Sensor and WiFi. University of Electronic Science and Technology, 2018.

[5] Manos, A.-Klein, I.-Hayan, T.: Gravity-Based Methods for Heading Computation in Pedestrian Dead Reckoning. Sensors (Basel), Vol. 19, 2019, No. 5, Art. No. 1170, 19 pp., doi: 10.3390/s19051170.

[6] Kang, J.-Lee, J.-Eom, D.-S.: Smartphone-Based Traveled Distance Estimation Using Individual Walking Patterns for Indoor Localization. Sensors (Basel), Vol. 18, 2018, No. 9, Art. No. 3149, 18 pp., doi: 10.3390/s18093149. 
[7] Lu, C.-Uchiyama, H.-Thomas, D.-Shimada, A.-Taniguchi, R. I.: Indoor Positioning System Based on Chest-Mounted IMU. Sensors (Basel), Vol. 19, 2019, No. 2, Art. No. 420, 20 pp., doi: 10.3390/s19020420

[8] Hasan, M. A.-Mishuk, M. N.: MEMS IMU Based Pedestrian Indoor Navigation for Smart Glass. Wireless Personal Communications, Vol. 101, 2018, pp. 287-303, doi: $10.1007 / \mathrm{s} 11277-018-5688-3$

[9] Jimenez, A. R.-Seco, F.-Prieto, C.-Guevara, J.: A Comparison of Pedestrian Dead-Reckoning Algorithms Using a Low-Cost MEMS IMU. 2009 IEEE International Symposium on Intelligent Signal Processing, Budapest, Hungary, 2009, pp. 37-42, doi: 10.1109/WISP.2009.5286542.

[10] Ibarra Bonilla, M. N.-Escamilla-Ambrosio, P. J.-Ramírez CorTÉs, J. M.: Pedestrian Dead Reckoning Towards Indoor Location Based Applications. $20118^{\text {th }}$ International Conference on Electrical Engineering, Computing Science and Automatic Control, Merida City, Mexico, 2011, 6 pp., doi: 10.1109/ICEEE.2011.6106608

[11] Basiri, A.-Lohan, E. S.-Moore, T. et al.: Indoor Location Based Services Challenges, Requirements and Usability of Current Solutions. Computer Science Review, Vol. 24, 2017, pp. 1-12, doi: 10.1016/j.cosrev.2017.03.002.

[12] Phan, A. V.-Nguyen, M. L.-Bui, L. T.: Feature Weighting and SVM Parameters Optimization Based on Genetic Algorithms for Classification Problems. Applied Intelligence, Vol. 46, 2017, No. 2, pp. 455-469, doi: 10.1007/s10489-016-0843-6

[13] Wang, W.-LiU, X. M.-Li, M. Z.-Wang, Y.B.-Wang, C. H.: Optimizing Node Localization in Wireless Sensor Networks Based on Received Signal Strength Indicator. IEEE Access, Vol. 7, 2019, pp. 73880-73889, doi: 10.1109/ACCESS.2019.2920279.

[14] WAng, H.: Implementation of RSSI-Based Localization Algorithm in Wireless Sensor Network. Beijing University of Posts and Telecommunications, Vol. 8, 2010, pp. 26-27.

[15] Lin, S. W.-Ying, K. C.-Chen, S. C.-Lee, Z. J.: Particle Swarm Optimization for Parameter Determination and Feature Selection of Support Vector Machines. Expert Systems with Applications, Vol. 35, 2008, No. 4, pp. 1817-1824, doi: 10.1016/j.eswa.2007.08.088

[16] Song, J.-Xu, Y.-LiU, Y.-Zhang, Y.: Investigation on Estimator of Chirp Rate and Initial Frequency of LFM Signals Based on Modified Discrete Chirp Fourier Transform. Circuits, Systems, and Signal Processing, Vol. 38, 2019, No. 12, pp. 58-61, doi: 10.1007/s00034-019-01171-5. 


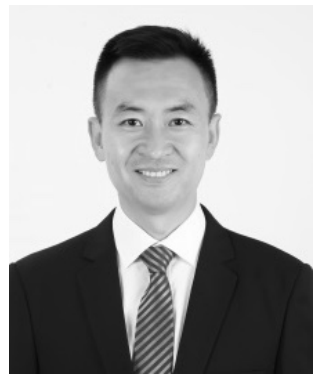

Wei WANG received his B.Sc. degree from China University of Mining and Technology, Xuzhou, Jiangsu, China, in 2002, the M.Sc. degree from North University of China, Taiyuan, Shanxi, China, in 2007, and the Ph.D. degree from Taiyuan University of Technology, Taiyuan, Shanxi, China, in 2011. His research interest includes wireless sensor networks and signal processing, fundamental study of WSN node location, and intelligent algorithms. Since 2016, he has served as Assistant Professor in the Information and Communication Engineering Department, North University of China. He is the author of one book and more than 20 articles.

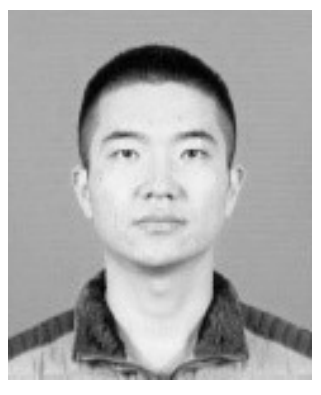

Cunhua WANG received his B.Eng. degree from North University of China. He is currently pursuing the M.Sc. degree with the North University of China. His research interests include signal processing and magnetic field indoor positioning.

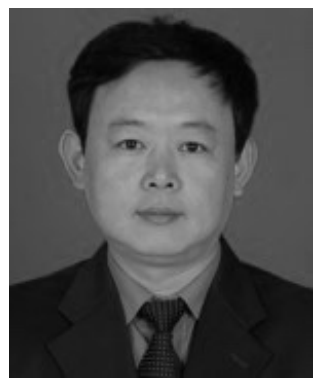

Zhaoba WANG is Professor in the Department of Information and Communication Engineering, North University of China. He received his Ph.D. degree from the Detection Technology, Nanjing University of Science and Technology in 2002. His main research interests include signal and information processing. He is the author of more than 80 articles.

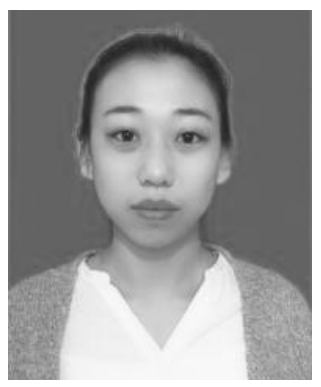

Xiaoqian ZHAo received her B.Eng. degree from Xinzhou Teachers University. She is currently pursuing her M.Sc. degree with the North University of China. Her research interests include signal processing and magnetic field indoor positioning. 